\title{
Equilibrium Study of Natural Non Toxic Solvents for Recovery of Trans- Aconitic Acid by Reactive Extraction
}

\author{
R. P. Nimbalkar1, R. P. Ugwekar², S. K. Deshmukh³ \\ ${ }^{1}$ Assistant Professor, ${ }^{2}$ Associate Professor, ${ }^{3}$ Professor \\ 1,3Priyadarshini Institute of Engineering \& Technology, Nagpur, Maharashtra, India \\ ${ }^{2}$ Laxminarayan Institute of Technology, Nagpur, Maharashtra, India
}

\begin{abstract}
Trans-aconitic acid (Propene-1 2 3-tricarboxylic acid) is an organic acid and is generally found in sugar cane. It is used as accidulent in food industry and can be used in synthesis of plasticizers. It is widely used in tissue engineering application. The present work focused on extraction of Trans- aconitic acid from its aqueous solution by using Aliquat 336 using natural non toxic diluents like Sunflower oil, Groundnut oil and Rice bran oil. Physical extraction and chemical extraction has been done to find out equilibrium parameters such as distribution constant, degree of extraction, loading ratio and equilibrium complexion constant. The experimental results are distribution coefficient (sunflower oil (2.81), groundnut oil (2.80) and for Rice oil (2.92), degree of extraction ( 56 to $81 \%$ for sunflower oil, 59 to $86 \%$ for groundnut oil, 58 to $82 \%$ for Rice bran oil), loading ratio (< 0.5 ) and equilibrium complexation constant $1.1152 \mathrm{l} / \mathrm{gm}$ equi for Sunflower oil, 1.6128l/gm equi for groundnut oil and 4.5556 l/gm equi for Rice Bran Oil. Water co extraction has also been studied, not more than $3 \%$.
\end{abstract}

\section{KEYWORDS: Trans-Aconitic acid, Aliquat-336, non-toxic diluents, distribution coefficient, degree of extraction}

\section{INTRODUCTION}

Aconitic acid (Pro-1 -ene 123 tricarboxylic acid) is used in food industry as an accidulent and also used for synthesis of biodegradable polyster. Commercially it can be prepared by dehydration of citric acid using sulphuric acid by thermal dehydration. Aconitic acid is also prepared by propogating aspergillus itaconicus or aspergillus terreus under aerobic condition from sugar cane juice or by molasses. But commercial manufacturing by fermentation is not possible. Thus reactive extraction of carboxylic acid is found to be a convenient method for recovery of caboxylic acid. Aconitic acid having two isomers as cis-aconitic acid and transaconitic acid. Cis-aconitic acid is formed in Krebs tricarboxylic acid cycle. ${ }^{[1]}$ Trans-aconitic acid is more stable form of carboxylic acid. Trans-Aconitic acid is white crystalline powder. It is odourless, solubility of aconitic acid in water is $5.5 \mathrm{~mL} / \mathrm{g}$ at $13^{\circ} \mathrm{C}$.Aconitic acid is a potential source of biodegradable plastic which can be used as a structure for skin and bone tissue engineering. ${ }^{[2]}$

Reactive extraction of carboxylic acid using different dilutents like aliphatic hydrocarbon and aromatic hydrocarbon was carried out by various researchers. ${ }^{[3-7]}$ Some researchers discussed recovery of carboxylic acid from fermentation broth and waste water. ${ }^{[7-10]} \mathrm{A}$ major drawback of fermentation to produce carboxylic acid is recovery of these acids from dilute solutions where they formed. The main problem of fermentation is the that when acid generates PH of the system falls (Waghmare 2011)

The reactive extraction has found to be a promising method for the recovery of the carboxylic acids from fermentation broth. As reactive extraction found to be effective at high concentration of substrate in the extractive fermentation, acid can be re-extracted and the solvent can be reused, better control of $\mathrm{pH}$,Better recovery of acid with higher product purity ,reduction of downstream processing load and recovery cost, reactants are relatively immiscible, reaction products to be separated are immiscible with the reaction phase, phase equilibrium can be positively influenced, heat transfer is to be improved during the reaction, product-catalyst separation can be affected by a liquid-liquid separation. Reactive extraction represents a reaction between the acid (solute) and extractant molecule at the interphase of aqueous and organic phase where transfers of acid molecules takes place by the diffusion and solubilization mechanism. Reactive extraction strongly depends on various parameters such as aqueous phase composition, organic phase composition, types of complexes (1:1, 2:1, etc.) formed, properties of the solvent (extract ant and diluent), type of solvent, temperature and $\mathrm{pH}$. Many authors tried to recover carboxylic acid by physical extraction i.e.by pure solvent but results were very poor. This may be because of high affinity towards water. Hence chemical extraction by using Organophosphorous compounds and secondary, tertiary and qua-ternary amine were tried and found suitable for extraction.

A study was carried out by Jun et al.( 2007), where it was observed that the reaction rates were affected by $\mathrm{pH}$ and contamination present in the aqueous phase. At a pH greater than the $\mathrm{pK}_{\mathrm{a}}$ of acid, more dissociation took place leading to the reduction in the extraction efficiency. Therefore,it was recommended for effective separation of acid from the production media, the $\mathrm{pH}$ of the fermentation broth should be kept at a value less than the $\mathrm{pK}_{\mathrm{a}}$ of the acid. Keshav A. et al (2012) Use a cheap, nontoxic, and selective solvents ,economic for the recovery of carboxylic acids produced by the bioroute.Solved problems for the recovery of the acid ([H3A $\left.] \mathrm{aq}^{0} 0.1-0.8\right)$ was solved by using tri-n-octylamine in natural diluents like rice bran oil, sunflower oil, soybean oil, and sesame oil .TOA was found to be most effective in removal of acid .Wasewar K. et al (2010) found out that reactive extraction is a promising method to recover carboxylic acid but suffers from toxicity problems of the 
diluents using a natural non-toxic solvents and extractant. Jaquet A. et al (1999) use extraction technique by using Aliquat 336, dissolved in octan-1-ol showed effect of molecular toxicity on the growth of Pseudomonas putida and concluded a strict control of the $\mathrm{pH}$ for cultivation must 7.47.5 for the growth. Waghamre et al (2011) presented work on extraction of Picolinic acid using tri-n-butyl phosphate (TBP) in sunflower oil and castor oil.

No work was found out on extraction of Trans aconitic acid by using non toxic diluents with Alamine 336 as extractant. Thus the present work focused on extraction of Trans Aconitic acid , the influence of non toxic diluents and effect on equilibrium extraction has been studied. Physical extraction and chemical extraction experiments were conducted and results have been found out.

\section{EXPERIMENTAL:}

Aconitic acid, (C6H606, trans-propene-1, 2, 3,-tri carboxylic acid), has molecular weight $174 \mathrm{~g} / \mathrm{mol}$ is an unsaturated tri basic aliphatic acid, also known as TAA, is a white to yellowish crystalline solid, with melting point $195{ }^{\circ} \mathrm{C}$. Its Hydrogen bond donor count is 3 whereas its Hydrogen bond acceptor count is 6 . It is soluble in water and alcohol. Its solubility in water increases from $18.6 \mathrm{~g} / 100 \mathrm{~mL}$ at $13^{\circ} \mathrm{C}$ to $110.7 \mathrm{~g} / 100 \mathrm{~mL}$ at $90^{\circ} \mathrm{C}$ (Patarau, 1989). Trans-Aconitic Acid used here is $98 \%+$ pure, supplied by Sigma Aldrich. Natural solvents were used for extraction and they were sunflower oil, groundnut oil and rice bran oil. The oils were obtained from local market and were used without water .Aliquat 336(9-octanoyl-8,10-dioxoheptadecan-9-yl) has molecular weight $446.113 \mathrm{~g} / \mathrm{mol}$. It is a quaternary ammonium salt used as a phase transfer catalyst and metal extraction reagent. It contains a mixture of C8 (octyl) and C10 (decyl) chains with C8 predominating. ammonium salt used as a phase transfer catalyst and metal extraction reagent. Hydrogen Bond Donor Count is 1, where as Hydrogen Bond Acceptor Count is 4 . It is yellowish orange in colour. Its density is $0.884 \mathrm{~g} / \mathrm{cm}^{3}$. It is highly viscous with coefficient of viscosity $1500 \mathrm{mPa} . \mathrm{s}$ at $30^{\circ} \mathrm{C}$ Aliquat 336 (methyltricaprylammonium chloride) is procured from Himedia, India and it is $99 \%$ pure. $\mathrm{NaOH} \&$ Phenolphthalein for titration \& Oxalic acid for standardizing $\mathrm{NaOH}$ are obtained from Fischer Scientific Pvt. Ltd.

Experiments were carried out by shaking equal volumes of (15ml) of aqueous and organic phases for $8 \mathrm{~h}$. at constant temperature $(305 \mathrm{~K})$ in a shaker. After shaking the phases were allowed to separate in separating funnel for $2 \mathrm{~h}$. Aqueous phase ${ }_{\mathrm{PH}}$ meter 0 . Acid concentration is in the range of $0.028717 \mathrm{~mol} \cdot \mathrm{L}-1-0.22974 \mathrm{~mol} \cdot \mathrm{L}-1$.Aliquat 336 extractant was used $20 \%$ and $40 \%$ with Sunflower oil, groundnut oil and rice bran oil as diluents.

The extraction ability was represented by the distribution coefficient. The calculated distribution coefficient KD, is calculated with following relation as given by following

$$
\mathrm{K}_{\mathrm{D}}=[\mathrm{HA}]_{\mathrm{org}} /[\mathrm{HA}]_{\mathrm{aq}}
$$

Where, $\mathrm{C}_{\text {org }}$ is the concentration of Trans-aconitic acid in organic phase and $\mathrm{C}_{\mathrm{aq}}$ is the concentration of Trans-aconitic acid in aqueous phase after reaching equilibrium. Degree of extraction (E \%) is defined as the ratio of Trans-Aconitic acid concentration in organic phase to the sum of acid concentration in organic and aqueous phase and is defined in term of $K_{D}$ as

$$
E \%=K_{D} \times 100 /\left(1+K_{D}\right)
$$

\section{REULTS \& DISCUSSION :}

The equilibrium study of physical and chemical extraction of Trans Aconitic acid from aqueous streams with sunflower oil, groundnut oil \& Rice Bran oil was studied. The extractant used for chemical extraction was (9-octanoyl-8, 10dioxoheptadecan-9-yl) azanium chloride, i.e. Aliquat 336. The objectives of this work were as follows

1. To study the equilibrium physical \& chemical extraction of Trans Aconitic acid from the aqueous stream.

2. To calculate the Distribution coefficients and Extraction efficiencies for various concentration combinations of aqueous acid and extractant Aliquat 336.

3. To calculate the complexation constant K11 for Aliquat 336 concentrations of $20 \%$ \& $40 \%(\mathrm{~V} / \mathrm{V})$.

4. To study the water co-extraction.

\subsection{PHYSICAL EXTRACTION:}

The physical extraction of Trans-Aconitic acid was carried using sunflower oil, groundnut oil \& Rice Bran oil. The plot of concentration of organic phase to that of aqueous phase is shown in Figure 3.1 Groundnut oil showed the best results among all the solvents, followed by Sunflower oil and Rice bran oil. The maximum extraction was obtained using Groundnut oil. The overall extraction efficiency for Groundnut oil, Sunflower oil \& Rice Bran oil was found to be 45.01606, 24.84658 and 18.74286.

The results of the physical extraction are tabulated in Table 3.1. It can be observed that the acid transferred to organic phase \& thus the distribution coefficient and the extraction efficiency increases with the aqueous TAA concentration. The maximum values of the distribution coefficient \& extraction efficiencies were observed for the aqueous acid concentration of $0.689219 \mathrm{~N}$; which was the highest concentration of aqueous acid used during equilibrium studies. The maximum values of Distribution coefficients observed at aqueous acid concentration of $0.689219 \mathrm{~N}$; which are 1.312817, 0.42289 and 0.351093 for groundnut oil, sunflower oil \& rice bran oil.

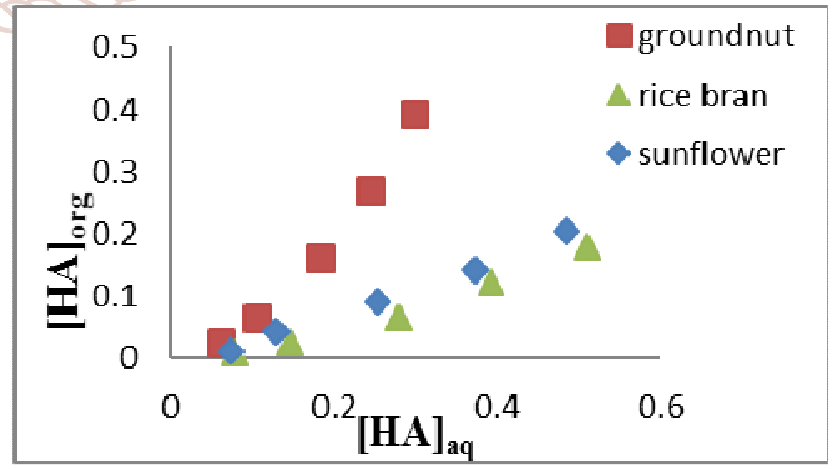

Figure.3.1..Physical extraction of Trans Aconitic acid using Groundnut oil, Sunflower oil, \& Rice Bran oil

\subsection{CHEMICAL EXTRACTION}

The chemical extraction of Trans-Aconitic acid was carried using Groundnut oil, Sunflower oil, \& Rice Bran oil. Aliquat 336 was used as the extractant with $20 \%$ \& $40 \%$ (V/V) concentration. For $20 \%$ Aliquat 336, the plot of concentration of organic phase to aqueous phase is shown in Figure 3.2. Rice bran oil showed the best results among all the solvents, followed by Sunflower oil and Groundnut oil, for $20 \%$ Aliquat 336 concentration. The overall extraction 
efficiency for Sunflower oil, Groundnut oil \& Rice Bran oil was found to be $67.87671 \%, 68.5538 \%$ \& $66.49108 \%$.

The results of chemical extraction with $20 \%$ Aliquat 336 concentration are tabulated in Table 3.2. It can be observed that the acid transferred to organic phase \& thus the distribution coefficient and the extraction efficiency increases with the aqueous acid concentration. The maximum values of the distribution coefficient \& extraction efficiencies were observed for the aqueous acid concentration of $0.689219 \mathrm{~N}$; which was the highest concentration of aqueous acid used during equilibrium studies. The maximum values of Distribution coefficients observed at aqueous acid concentration of $0.689219 \mathrm{~N}$; for Sunflower oil, Groundnut oil and Rice bran oil are 2.813109, $2.807842 \& 2.927176$ respectively.

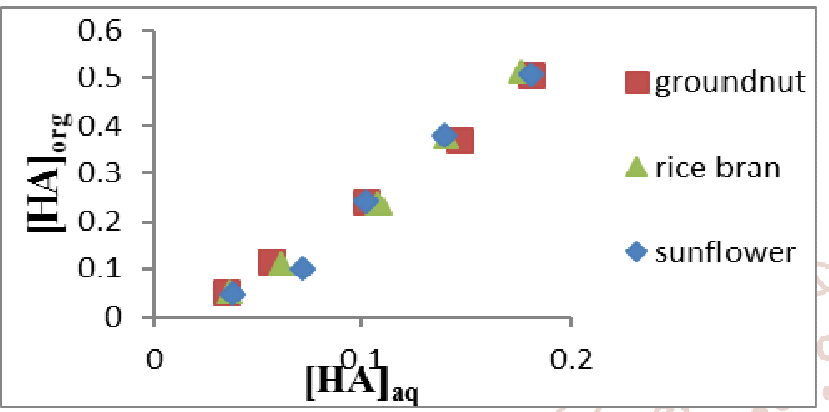

Figure 3.2: Chemical extraction of Trans Aconitic acid with $20 \%$ TBP (V/V) using Groundnut oil, Sunflower oil \& Rice Bran oil.

For $40 \%$ Aliquat 336 loaded organic phase, the plot of concentration of organic phase to aqueous phase is shown in Figure 3.3 Groundnut oil showed the best results among all the solvents, followed by Rice Bran oil \& Sunflower oil. The overall extraction efficiency for Groundnut oil, Rice Bran oil \& Sunflower oil was found to be $81.29285,80.96397$ \& 78.41761. The results of chemical extraction with $40 \%$ Aliquat 336 concentration are tabulated in Table 3.3.The maximum values of the distribution coefficient \& extraction efficiency was observed for the aqueous acid concentration of $0.689219 \mathrm{~N}$; which was the highest concentration of aqueous acid used during equilibrium studies. The maximum values of Distribution coefficients observed at aqueous acid concentration of $0.689219 \mathrm{~N}$; for Sunflower oil, Groundnut oil and Rice bran oil are 4.50275, 6.657994 \& 4.672588 respectively.

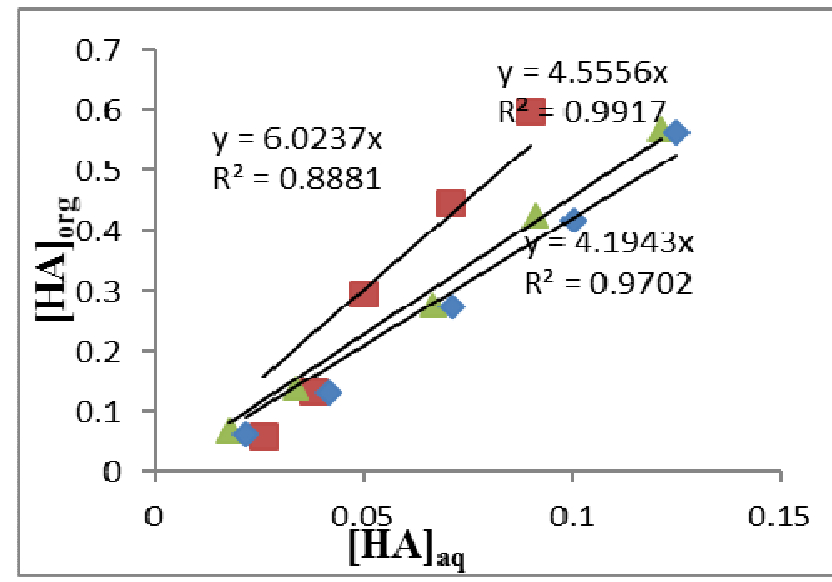

Figure 3.3: Chemical extraction of Trans Aconitic acid with $40 \%$ TBP (V/V) using Groundnut oil, Sunflower oil \& Rice Bran oil.
The stoichiometry of the overall extraction reaction is dependent on the loading ratio in the organic phase, $\mathrm{z}$. If the organic phase is not highly concentrated, i.e., at very low loading ratios $(\mathrm{z}<0.5)$, the $1: 1$ complex is formed and a plot of $\mathrm{z} /(1-\mathrm{z})$ versus $[\mathrm{HA}]_{\mathrm{aq}}$ is a straight line whose slope gives the complexation constant, $\mathrm{K}_{11}$.

$$
\frac{Z}{1-z}=K_{11} \times[H A]_{a q}
$$

A straight line of the plot of equation above is shown in Figure 3.4. The extractant concentration used was $20 \%$ (V/V) Aliquat 336 using Groundnut oil, Sunflower oil \& Rice bran oil with a slope of $1.5382,1.5309 \& 1.5681$ respectively. Hence, the equilibrium complexation constant for the 1:1 acid: extractant complex at $25^{\circ} \mathrm{C}$ for the extraction of Trans Aconitic acid with Aliquat 336 dissolved in Groundnut oil, Sunflower oil \& Rice Bran oil, for low concentrations of Trans Aconitic acid in the organic phase, is 1.5382 lit/gram eq. for Ground nut oil ,1.5309 lit/gram eq. for Sunflower oil \& 1.5681 lit/gram eq. for Rice bran oil.

Similarly, The complexation constant for the 1:1 acid: extractant complex at $25{ }^{\circ} \mathrm{C}$ for the extraction of Trans Aconitic acid with Aliquat 336 dissolved in Groundnut oil, Sunflower oil \& Rice Bran oil, for low concentrations of Trans Aconitic acid in the organic phase, is 1.6128 lit/gram eq. for Groundnut oil,1.1152 lit/gram eq. for Sunflower oil \& 4.5556 lit/gram eq. for Rice Bran oil .

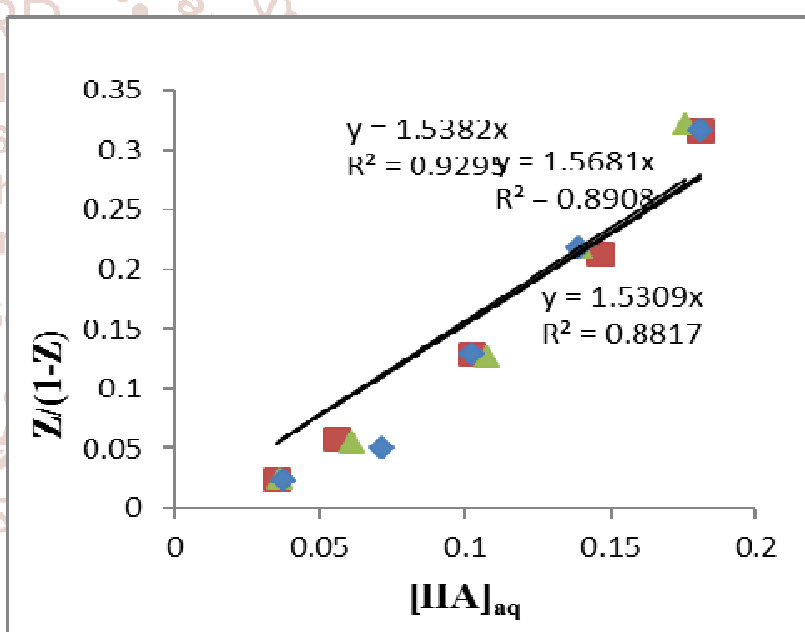

Figure 3.4: $\mathrm{K}_{11}$ for $20 \%(\mathrm{~V} / \mathrm{V}$ ) Aliquat 336 as extractant using Groundnut oil, Sunflower oil \& Rice Bran Oil.

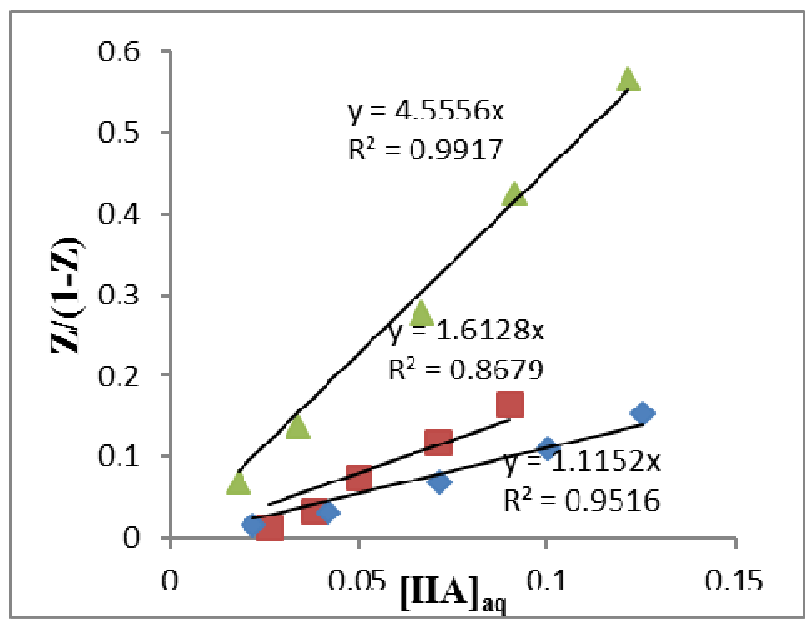

Figure 3.5: $\mathrm{K}_{11}$ for $40 \%(\mathrm{~V} / \mathrm{V})$ Aliquat 336 as extractant using Groundnut oil, Sunflower oil \& Rice Bran Oil. 


\subsection{Water Co-extraction:}

The solubility between organic and aqueous phases depends on the characteristics of the system species, their concentrations, temperature, and other process conditions. Trans Aconitic acid is a weak acid and mutual solubility may cause significant change in volume. The volume change of the organic phase can be associated with water coextraction. In many applications of carboxylic acid, pure acid is required, and water co-extraction has extra expenses on purification and hence the overall economics. ${ }^{37}$ The water co-extraction experiments were carried out for Trans Aconitic acid-Aliquat 336 -diluents (groundnut oil, sunflower oil \& rice bran oil). Different concentrations of Trans Aconitic acid and Aliquat 336 were used for $20 \mathrm{~mL}$ of each initial phase volume. Table (4.1, 4.2, and 4.3) depicted the experimental data for water co-extraction. The volume of the organic phase was increased slightly with an increase in the acid concentration for $20 \%$ Aliquat 336 in diluents. The volume of organic phase was increased and almost constant for all concentrations of acid in all cases for $40 \%$ TBP in diluents. The diluents play a significant role for water coextraction. At low concentration of Aliquat 336 in diluents, more water is co-extracted. For higher solvation of complex in diluents, more water co-extraction is provided in the case of higher acid concentrations and opposite for low concentration of acid. Wasewar et al. (2011) Waghmare et al (2011) and Keshav et al. (2009) observed the similar kind of results for extraction of itaconic acid, picolinic acid, and propionic acid. However, the carboxylic acids selectivity is very high over water as compared to only conventional diluents. Below 3\% water co-extraction in reactive extraction of Trans Aconitic acid by Aliquat 336 in different diluents was observed. This water co-extraction may have a much less effect on process feasibility.

\section{CONCLUSIONS:}

The removal of aqueous phase Trans Aconitic acid by reactive extraction using Aliquat 336 in Groundnut oil Sunflower oil and Rice Bran oil was studied. The results are presented in terms of various physical and chemical extraction equilibrium parameters. Less than 0.5 loading was observed and only 1:1 Trans Aconitic acid-Aliquat 336 complex in Groundnut oil, Sunflower oil and Rice Bran oil was formed. Significant enhancement in removal was observed by reactive extraction. Extraction efficiency up to $86 \%$ was observed. The Distribution coefficients for physical \& chemical extraction were calculated for all the three solvents. Equilibrium constants were also calculated for $20 \%$ and $40 \%$ Aliquat 336 concentrations for all the solvents. The preference of diluents were observed as Groundnut oil > Sunflower oil > Rice Bran oil, for the extraction of Trans Aconitic acid. Water co-extraction was also studied, not more than $3 \%$ water co-extraction was found.

\section{REFERENCES:}

[1] Kertes, A. S.; King, C. J., "Extraction Chemistry of Fermentation Product Carboxylic Acids" Biotechnol. Bioeng. 1986, 28, 269

[2] Akanksha Kanitkar et al , "In vitro characterization of polyesters of aconitic acid, glycerol, and cinnamic acid for bone tissue engineering",J Biomater Appl March 2015 vol. 29 no. 8 1075-1085
[3] S.Kumar et al, Intensification of Nicotinic Acid Separation using Organophosphorous Solvating Extractants by Reactive Extraction, Chemical Engineering \& Technology, Volume 31, Issue 11, November, 2008, Pages 1584-1590.

[4] A. Keshav et al, "Reactive Extraction of Propionic Acid Using Tri-N-butyl Phosphate in Petroleum Ether: Equilibrium Study" Chemical and biochemical engineering quarterly, Vol.22 No.4 Prosinac 2008

[5] YK Hong et al, "Reactive extraction of lactic acid with mixed tertiary amine extractants", Biotechnology Techniques, December 1999, Volume 13, Issue 12, pp 915-918.

[6] Kailas L. Wasewar et al, "Reactive extraction of lactic acid using alamine 336 in MIBK: equilibria and kinetics", Journal of Biotechnology, Volume 97, Issue 1, 17 July 2002, Pages 59-68

[7] Amit Keshav, "Recovery of propionic acid from aqueous phase by reactive extraction using quarternary amine (Aliquat 336) in various diluents" ,Chemical Engineering Journal Volume 152, Issue 1, 1 October 2009, Pages 95-102

[8] Bong Seock Kim, "Effect of salts on the extraction characteristics of succinic acid by predispersed solvent extraction",Bioprocess Eng. (2004) 9: 207.

[9] Julio Berrios, "Gibberellic acid extraction from aqueous solutions and fermentation broths by using emulsion liquid membranes", Journal of Membrane Science, Volume 348, Issues 1-2, 15 February 2010, Pages 9198 ilc

[10] V. F. Pfeifer, "Itaconic Acid by fermentation with Aspergillus Terrus", Ind. Eng. Chem., 1952, 44 (12), pp 2975-2980

[11] Mangesh D. Waghmare et.al., "Natural Nontoxic solvents for recovery of Picolinic acid by Reactive Extraction", Ind. Eng. Chem/Res.,2011,50,1352613537.

[12] Kailash L. Wasewar et al, "Reactive Extraction of itaconic acid using tri-n-butyl phosphate and aliquat 336 in Sunflower oil as a non toxic solvents",J Chem Technol Biotec hnol,2011,86,319-323.

[13] Amit Keshav et.al, "Reactive extraction of Acrylic acid using Tri-n-butyl Phosphate in different Diluents" ,J. Chem. Eng. Data , 2009,54,1782-1786.

[14] Paturau, J. M, "By-products of the cane sugar industry. An introduction to their industrial utilization",1989

[15] Kanti K. Athankar et al , "Reactive extraction of Phynylacetic acid with Tri-n-butyl Phosphate in Benzene, Hexanol and Rice bran Oil at 298 K", J. Chem. Eng. Data ,2013,58,3240-3248

[16] Dipaloy Datta and Sushil Kumar, "Reactive Extraction of Pyridine-2-carboxylic acid (Picolinic Acid)Using Nontoxic Extractant and diluents systems", J. Chem. Eng. Data. 